Check for updates

Cite this: RSC Adv., 2017, 7, 36313

\title{
Rapid spray-crosslinked assembly of a stable high- performance polyelectrolyte bipolar membrane
}

\author{
Wen Zhu, $\uparrow$ Haizhi Wang, $\uparrow$ Guoshan Jing, Qianqian Liu, Chunxi Li $\mathbb{D}$ \\ and Hong Meng (DD *
}

Bipolar membrane processes have evolved as one of the most promising technologies in the field of acid and base production as well as many interesting separation applications. Herein, we report a rapid spraycrosslinked assembly for fabricating a high-performance polyelectrolyte bipolar membrane. Polyvinyl alcohol (PVA), glutaricdialdehyde (GA) and polyethyleneimine (PEI) are alternatively and successively sprayed onto a supporting cation-exchange membrane. GA crosslinked PVA and PEI are responsible for the interlayer and anion-exchange layer, respectively. Under optimal conditions, at a current density of $100 \mathrm{~mA} \mathrm{~cm}{ }^{-2}$, the cell voltage of the BPM was as low as $1.79 \mathrm{~V}$. Moreover, it has been demonstrated that the spray crosslinked membrane has good stability in acid and alkali harsh conditions. In contrast to the traditional casting method, spray assembly combined with chemical cross-linking can tune the membrane thickness at the nanoscale and microscale levels, as well as increase its charge density and ion-exchange capacity. This facile approach might open a new way to efficiently and flexibly produce various bipolar membranes with different polyelectrolytes.

Received 12th May 2017

Accepted 4th July 2017

DOI: $10.1039 / c 7 r a 05367 c$

rsc.li/rsc-advances membranes materials for the anion- and cation-exchange layers of BPMs. ${ }^{13}$

Currently, most of the BPMs are normally produced by direct adhering of commercial anion- and cation-exchange membranes together with hot-pressure and casting a cation (or an anion) exchange membrane onto the oppositely charged (anion or cation) membrane. ${ }^{\mathbf{1 4 , 1 5}}$ This process generally includes polymer dissolution, casting/coating onto substrate, solvent volatilization and post-crosslinking, which is a relatively complicate, time-consuming and highly inefficient. ${ }^{\mathbf{1 6}}$ More importantly, it is difficult to tune the intermediate layer on the nanometer scale. Therefore, the reported intermediate layer is relatively thick at the micrometer scale. ${ }^{17-19}$ In recent years, spray assembly has attracted considerable attention in the field of membrane preparation, because it offers the advantages of mild membrane-forming conditions, controllability of thickness within nanometer scale. For example, Zhang ${ }^{20}$ had reported that the simultaneous spray self-assembly for the fabrication of a well-dispersed ZIF-8-PDMS nanohybrid membrane with an extremely high loading. It was demonstrated that the thickness in this study was far thinner than that of other reported butanol-permselective composite membranes. ${ }^{21}$ More recently, they also proposed to construct a super hydrophilic water uptake layer by spray-assisted biomineralization of calcium carbonate $\left(\mathrm{CaCO}_{3}\right)$ onto a (poly(acrylic acid)/ poly(ethyleneimine $))_{n} /$ polyacrylonitrile $\quad\left((\mathrm{PAA} / \mathrm{PEI})_{n} / \mathrm{PAN}\right)$ membrane. ${ }^{22}$ This work suggested that rapid spray strategy could not only tune the thickness but also could facilely tune the membrane surface morphology and wettability. However,
College of Chemical Engineering, Beijing University of Chemical Technology, Beijing 100029, China. E-mail: menghong@mail.buct.edu.cn; Fax: +86-10-64410308; Tel: $+86-10-64444911$

$\dagger$ These authors contributed equally to this study and share first authorship. 
these works particularly focused on the alcohol permselective membrane, none of work have dealt with the BPM using spray assembly.

In the present work, we proposed a rapid spray assembly coupled with chemical crosslinking to prepare a stable highperformance polyelectrolyte bipolar membrane. This technique was based on the ionization properties of the polyelectrolyte and the advantages of spraying assembly. In order to improve ion migration rate, promote water splitting, lower the IR drop, polyethyleneimine (PEI) was used to form the AM because PEI was a primary amine produced by polymerization of ethylene imine, branched polymer secondary and tertiary amines, in water to form a polycation, preparation of anion exchange membrane bipolar membrane layer materials was very good. ${ }^{23}$ Hydrophilic polyvinyl alcohol (PVA) as the interlayer because of its highly hydrophily, ${ }^{24}$ and commercial $\mathrm{CM}$ as cation-exchange layer, PVA, glutaricdialdehyde (GA) (crosslinkers), a high-performance BPM could be rapidly fabricated. In this case, spraying glutaricdialdehyde between PVA and PEI can crosslink both PEI and PVA, which made the resulting bipolar membrane much more stable. The spray-crosslinked membranes were intensively characterized by scanning electron microscopy (SEM), Fourier-transform infrared (FTIR) spectroscopy, and thermo gravimetric analysis (TGA). The membranes were frozen in liquid nitrogen, broken, and then sputtered with gold before SEM analysis. The influences of assembly conditions on water-dissociation voltage were also investigated.

\section{Experimental}

\subsection{Materials}

The cation-exchange membrane (CM-ZHJ) were obtained from Hangzhou Grion Environmental Technology Co., Ltd. Zhejiang, China. The CM-ZHJ with strongly acid groups, $\mathrm{R}_{-} \mathrm{SO}_{3}{ }^{-}$were provided by Hangzhou Grion Environmental Technology Co., Ltd. The cation-exchange membrane had thickness $0.42 \mathrm{~mm}$, water content $40-55 \%$, exchange capacity $2.0 \mathrm{~mol} \mathrm{~kg}^{-1}$, area resistance $11 \Omega \mathrm{cm}^{-2}$, and selective penetration 93\%. Respectively, PEI (50\% aqueous solution) and PVA (PVA-1799) were purchased from Aladdin Corporation Ltd. Shanghai, China. Sodium sulfate, sodium chloride, sodium hydroxide, hydrochloric acid all of analytical grade were from Beijing Chemical Works, Beijing China. GA (GA, 25\%) was purchased from Tianjin Fu chen Chemical Reagents Factory, Tianjin China. Nafion membranes was bought from DuPont Corporation.

\subsection{Spray assembly system}

In order to achieve automatic control of the BPM preparation process, we developed a spray system for membrane selfassembly based on a logic-control program (PLC system; Fig. 1). The device was mainly composed of gas supply systems, spray systems, a carrier membrane, and a PLC system.

The equipment consisted of three identical atomizing nozzles with a nozzle diameter of $0.5 \mathrm{~mm}$, each connected to a pressure tank and a solenoid valve that controlled the opening

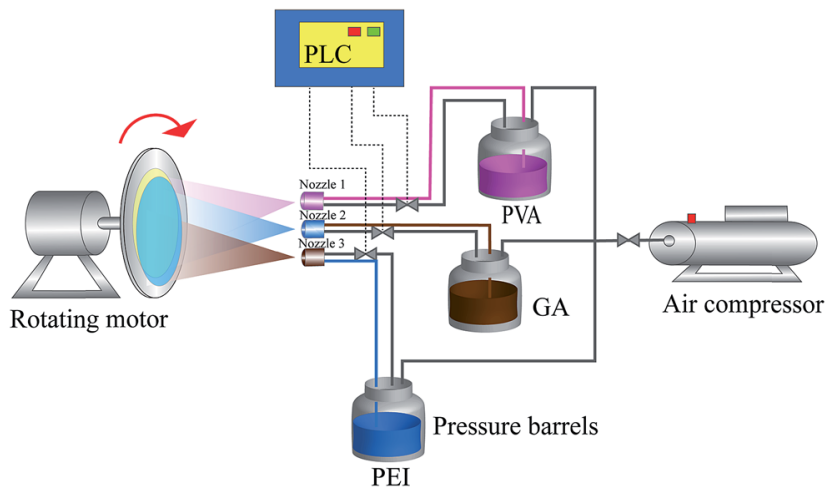

Fig. 1 Automatic spray system for the preparation of bipolar membranes.

and closing of the nozzles. A high volume air compressor was utilized to maintain a constant pressure of 2 bars inside the two pressure tanks while spraying. A metal substrate was fixed perpendicularly and rotated at $100 \mathrm{rpm}$, solely to accelerate the drainage of the solution from the surface of the membrane. The operating parameters, such as spraying time, standing interval, spray cycles, and on-off bunker, were controlled by a programmable logic controller (PLC) system. PLC also controlled sprayer and burner start and stop commands and allowed for fully automatic operation. The nozzles, the burner and the substrate were placed inside a Constant Temperature and Low Humidity Box (CTLH Box) which could be controlled at a constant temperature and humidity.

\subsection{Preparation of BPM}

The membrane preparation process was depicted in Fig. 2. PEI was dissolved in deionized water to form a $5 \mathrm{wt} \%$ PEI aqueous solution, which was stirred well to obtain a solution that formed a colorless transparent solution. A 3 wt \% PVA aqueous solution prepared from PVA was stirred in a $90{ }^{\circ} \mathrm{C}$ water bath for $1 \mathrm{~h}$ and then allowed to cool. GA ( $3 \mathrm{wt} \%)$ was mixed and stirred well. The $\mathrm{CM}-\mathrm{ZHJ}$, was cut into $5 \mathrm{~cm} \times 5 \mathrm{~cm}$ pieces, which were then soaked in distilled water for $24 \mathrm{~h}$. Subsequently, they were removed and fixed to the membrane carrier. A PVA aqueous solution (3 wt\%) was sprayed onto one topside of the CM-ZHJ and then $3 \mathrm{wt} \% \mathrm{GA}$ and $5 \mathrm{wt} \%$ PEI solutions were sprayed onto the same side. The spraying time and standing interval

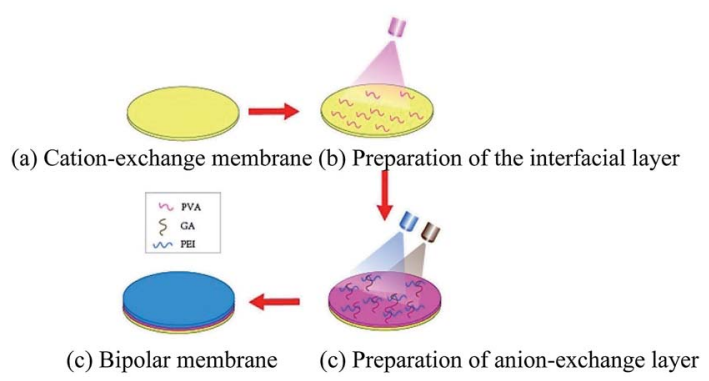

Fig. 2 Schematic of the preparation of the bipolar membrane. 
Table 1 The name abbreviations of the sample with different deposition condition

\begin{tabular}{ll}
\hline Abbreviations & Deposition condition \\
\hline CM-ZHJ & A commercial cation-exchange membrane \\
CM-ZHJ-PVA & Spraying 3\% PVA twice on CM-ZHJ \\
PEI/CM-ZHJ & Spraying 3\% PVA twice, 3\% GA three times, \\
& followed by 5\% PEI three times \\
$(\text { PEI-CYC/CM-ZHJ })_{1}$ & Spraying 3\% PVA twice, 5\% PEI and \\
& 3\% GA alternately one cycle \\
$(\text { PEI-CYC/CM-ZHJ })_{3}$ & Spraying 3\% PVA twice, and then 5\% \\
& PEI and 3\% GA alternately three cycle \\
$(\text { PEI-CYC/CM-ZHJ })_{5}$ & Spraying 3\% PVA twice, and then 5\% \\
& PEI and 3\% GA alternately five cycle
\end{tabular}

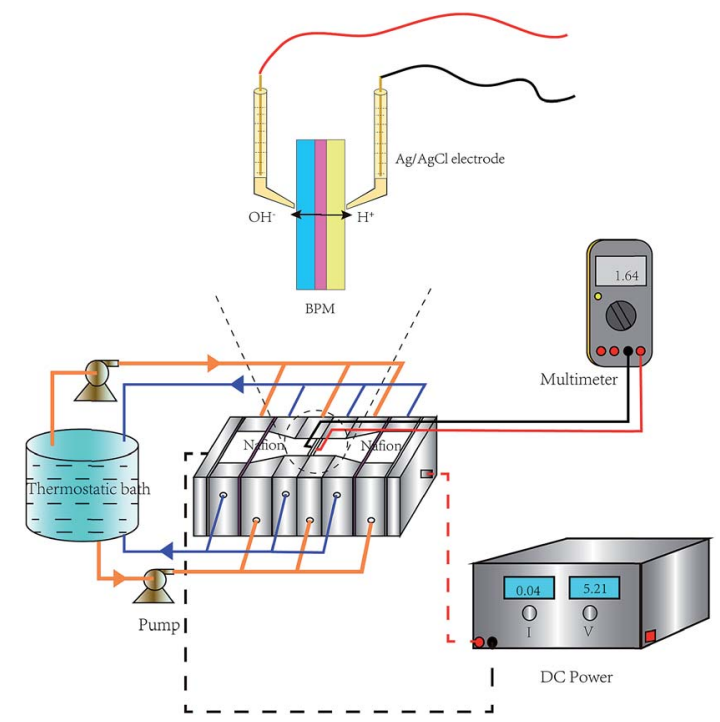

Fig. 3 Equipment for obtaining $I-V$ curves of the bipolar membranes.

between two spraying cycles was $5 \mathrm{~s}$ and 10 min respectively. Finally, the membrane was dried at room temperature to obtain BPM. The membranes with different deposition condition were denoted as CM-ZHJ, CM-ZHJ-PVA, PEI/CM-ZHJ BPM separately, which was shown in Table 1.

\subsection{Water dissociation on the BPM}

$I-V$ curves of a BPM could be used to determine its waterdissociation voltage, limiting current density, and watersplitting efficiency. ${ }^{25}$ The set used to obtain the $I-V$ curves of the BPM was shown in Fig. 3. Cathode and anode chambers were separated from the BPM by Nafion membranes to prevent electrode products from coming into contact with the BPM and to maintain the environments of the two compartments near BPM. The BPM was placed in the middle of the cell, and the trans-membrane voltages were measured by two $\mathrm{Ag} / \mathrm{AgCl}$ electrodes. The BPM specimen was immersed in the $\mathrm{Na}_{2} \mathrm{SO}_{4}$ solution to keep it wet and then placed in the measuring cell. Before the experiment, each chamber was circulated for $30 \mathrm{~min}$ to eliminate visible bubbles. ${ }^{26}$ The device was run for $20 \mathrm{~min}$ at a current density of $20 \mathrm{~mA} \mathrm{~cm}$ co $^{-2}$ to allow BPM to reach steady state. The applied current density was then increased from 0 to $120 \mathrm{~mA} \mathrm{~cm}{ }^{-2}$ at a rate of $10 \mathrm{~mA} \mathrm{~cm} \mathrm{~cm}^{-2}$ while the voltage was recorded. The results were reported as the average of three runs.

\subsection{Membrane characterization}

SEM was utilized to observe the cross-sections and surface of the BPMs. ${ }^{27}$ A Nicolet-560 FTIR spectrometer was used to obtain the spectra for CM-ZHJ, CM-ZHJ-PVA, and PEI/CM-ZHJ BPM. TGA measurements on PEI/CM-ZHJ BPM were performed with a DT-40 thermal analyzer. The temperature was increased from 20 to $600{ }^{\circ} \mathrm{C}$ at a heating rate of $10^{\circ} \mathrm{C} \mathrm{min}^{-1}$.

\section{Results and discussion}

\subsection{SEM, FTIR analyses}

SEM images of the cross-section and surface morphology of the BPM were displayed in Fig. 4. The cross-sectional morphology of the PEI/CM-ZHJ BPM, which had different composite layers of polyelectrolyte (Fig. 4), clearly had a boundary layer. The CMZHJ was sprayed PVA twice, followed by spraying the optimum concentration of $5 \%$ PEI and $3 \%$ GA alternately one cycle ((PEICYC/CM-ZHJ $\left.)_{1}\right)$, three cycles ((PEI-CYC/CM-ZHJ $)_{3}$ ) and five cycles ((PEI-CYC/CM-ZHJ $\left.)_{5}\right)$ respectively. The upper anionexchange layer was formed by cross-linking reaction among GA, PEI, and PVA. Its cross-section was smooth and dense. Its thickness increased with the increase in number of composite layers, indicating its dependence on the spraying assembly. The cross-section formed. The transition zone in which water dissociation occurs was the PVA interface. Since it was of nanometer thickness, it was not visible in Fig. 4. Although the cross-sectional morphology of BPM was distinct, its anion- and cation-exchange layers were in close contact, showing no bubbles in between them. The surfaces of the BPM anion- and cation-exchange layers (Fig. 4d) showed smooth morphology, with the former layer being smoother and tighter.

Fig. 5 shows the IR spectra of CM-ZHJ, CM-ZHJ-PVA, and PEI/ $\mathrm{CM}-\mathrm{ZHJ}$ membranes. The characteristic absorption of sulfonic acid group at $1190-1120 \mathrm{~cm}^{-1}$ was present in the spectrum of

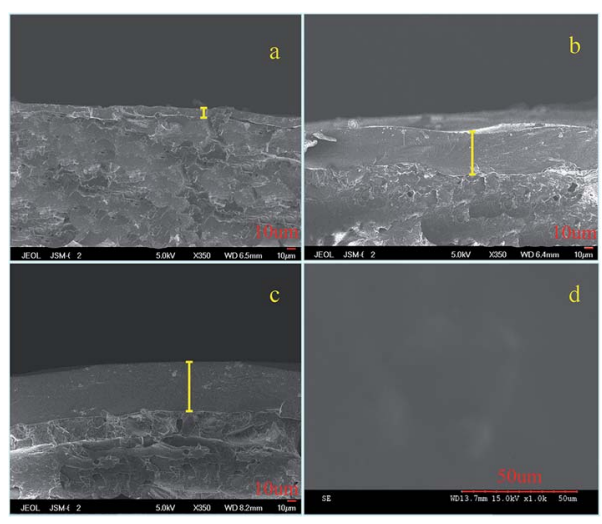

Fig. 4 SEM images for: (a) cross-section (PEI-CYC/CM-ZHJ) $)_{1}$ (b) cross-section (PEI-CYC/CM-ZHJ) ; (c) cross-section (PEI-CYC/CM$\mathrm{ZHJ})_{5}$; (d) Surface of $(\mathrm{PEI}-\mathrm{CYC} / \mathrm{CM}-\mathrm{ZHJ})_{3}$. 


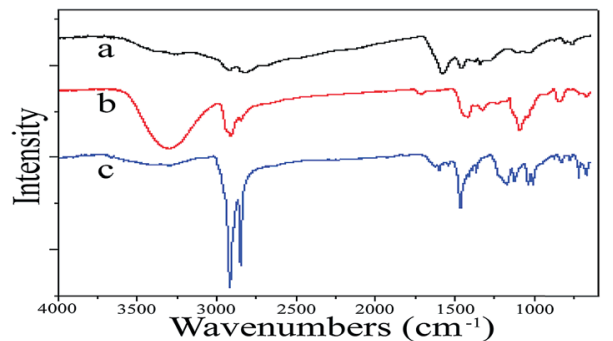

Fig. 5 FTIR spectra of the (a) PEI/CM-ZHJ bipolar membrane, (b) CMZHJ-PVA membrane, (c) CM-ZHJ membrane.

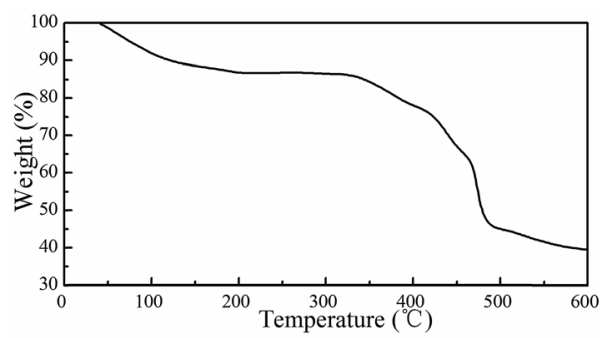

Fig. 6 TG profiles of the (PEI-CYC/CM-ZHJ) 3 bipolar membrane.

the CM-ZHJ membrane (Fig. 5c). The IR spectrum of the CMZHJ-PVA membrane, which was prepared by spraying an intermediate layer of PVA on the CM-ZHJ membrane (curve b is), had a broad band at $3600-3200 \mathrm{~cm}^{-1}$ due to $\mathrm{O}-\mathrm{H}$ stretching vibration. The absorption at $1091 \mathrm{~cm}^{-1}$ was due to $\mathrm{C}-\mathrm{O}$ stretching vibration..$^{28}$ The $\mathrm{O}-\mathrm{H}$ absorption peak in the FTIR spectrum of the BPM (curve a) was markedly weaker than that in curve b. The absorption peak at $1575 \mathrm{~cm}^{-1}$, which arised from $\mathrm{C}=\mathrm{N}$ stretching vibration, indicated that GA underwent cross-linking reaction with amide of PEI, generating imino groups and formed a dense membrane, thereby improving the mechanical properties of the membrane.

\subsection{Stability of spray-crosslinked membrane}

The thermal stability of the spray-crosslinked bipolar membrane was investigated by thermogravimetry (TG). The thermo gram of the PEI/CM-ZHJ BPM (Fig. 6) roughly had three sloping sections. The first section $\left(30-120^{\circ} \mathrm{C}\right)$, which showed a relatively small loss $(\sim 10 \%)$, corresponded to loss of water absorbed on the polymer membrane. The second section (100$\left.350{ }^{\circ} \mathrm{C}\right)$, which had a lower slope and loss $(\sim 5 \%)$, indicated the good thermal stability of the BPM within this temperature range. The slope of the third section was large and had a steep peak for the weight loss due to structural collapse of the sample, which caused $\mathrm{CO}_{2}$ release.

\subsection{Effects of assembly conditions on $I-V$ curves of the BPM}

3.3.1 Effect of PEI concentration. The effect of PEI concentration on the water-dissociation performance of the BPM was shown in Fig. 7. When the PEI concentration was low, the formative anion exchange layer was thin but relatively

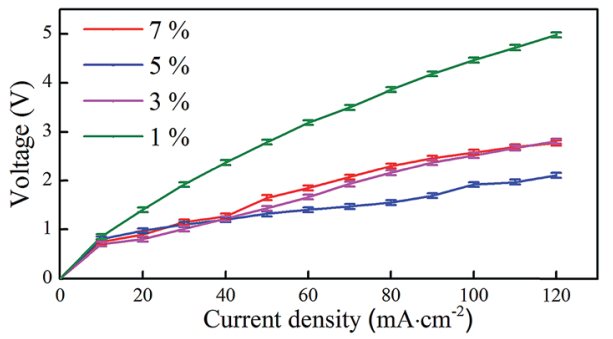

Fig. $7 \quad I-V$ curves of the bipolar membranes with anion-exchange layers prepared with different PEI concentrations.

homogenous. It possessed few fixed groups, thus impeding the transfer of $\mathrm{OH}^{-}$and causing a large water-dissociation voltage. With the increase in PEI concentration, the concentration of fixed groups increased and thereby enhanced the transfer of $\mathrm{OH}^{-}$. Because of the hydrophilicity of PVA, water migrated to the intermediate layer and splits. This reaction lowered the voltage across the BPM to a relatively stable level. Since its concentration of exchangeable groups was too high, the crosslinking reaction between layers was inhomogeneous. The electrostatic repulsion between layers was strong, the extension of hyper branched polymer chain segments was irregular, and the surface structure of the membrane gradually loosened when the PEI concentration was too high. Consequently, the voltage drop across the BPM rebounded during the testing process, and delamination or ballooning between layers easily occurred, thereby increasing the water-dissociation voltage and decreasing the mechanical stability of the membrane. When PVA $3 \mathrm{wt} \%$ solutions were sprayed twice and $2 \mathrm{wt} \%$ GA were sprayed three times, then, the appropriate concentration of PEI with molecular mass of 70000 sprayed three times was found to be $5 \mathrm{wt} \%$. The voltage across the BPM was $1.92 \mathrm{~V}$ (error \pm 0.05 ) at $100 \mathrm{~mA} \mathrm{~cm}{ }^{-2}$ current density. The voltage drop was small.

3.3.2 Effect of GA concentration. Since both PEI and PVA are water-soluble, GA was often cross-linked with them in order to increase the mechanical stability of membranes. The effect of the GA concentration on the water-dissociation voltage of the BPM was shown in Fig. 8. When the GA concentration was very low, both PVA and PEI gradually dissolved in water, since the cross-linking degree was not enough, resulting in less fixed groups in the layer and lower ion-exchange capacity. The crosslinking reaction was enhanced at higher GA concentration. Both PEI and PVA fixed on CM-ZHJ formed a dense anion-

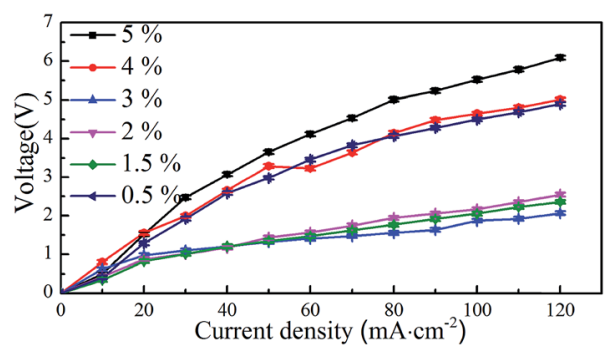

Fig. $8 \quad I-V$ curves of the bipolar membranes with anion-exchange layers prepared with different concentrations of GA solutions. 
exchange layer via cross-linking with GA, thus increasing the membrane stability. When PVA $3 \mathrm{wt} \%$ solutions were sprayed twice, the GA was sprayed three times, followed by the optimum concentration of PEI with molecular mass of 70000 sprayed three times, the membrane voltage increased because of excessive cross-linking, changing the membrane structure and affecting the water-dissociation voltage when the GA (spraying 3 times) concentration more than $3 \mathrm{wt} \%$. Thus, the PVA $3 \mathrm{wt} \%$ solutions were sprayed twice, $3 \mathrm{wt} \%$ GA were sprayed three times, and $5 \mathrm{wt} \%$ PEI with molecular mass of 70000 were sprayed three times, the optimum water-dissociation voltage of the BPM decreased from $1.92 \mathrm{~V}$ (error \pm 0.05 ) above result to $1.87 \mathrm{~V}$ (error \pm 0.05 ) at a current density of $100 \mathrm{~mA} \mathrm{~cm}^{-2}$.

3.3.3 Effect of PVA concentration. The effect of the PVA intermediate layer on the water dissociation on BPMs modified with 1, 2, 3, 4, and $5 \mathrm{wt} \%$ PVA solutions was examined from their $I-V$ curves (Fig. 9). The membranes was prepared with spraying PVA twice, the optimum concentration of $3 \%$ GA three times and 5\% PEI with molecular mass of 70000 three times. The curves showed that PVA at low concentration catalyzes water dissociation on the BPM. In contrast, PVA at high concentration was disadvantageous to the performance of the membranes. Thus, the water-dissociation voltage of the BPM decreased and then increased with the increase in PVA concentration. When the PVA concentration was low, the intermediate layer that formed was so thin that the surface of the anion- and cation-exchange layers came in and contacted with each other, resulting in failure of the BPM. Furthermore, hydrophilic groups in the intermediate layer were few and water dissociation was slow under this condition. The adsorbed amount also increased with the increase in PVA concentration. Therefore, the intermediate layer possessed more catalytic and hydrophilic sites, resulting in the observed water dissociation performance of the BPM. When the PVA concentration was too high, PVA molecules had many hydroxyl groups, which lead to strong interactions between PVA and water molecules and between PVA molecules. The intermolecular interaction greatly increased the thickness of the interfacial layer and formed a dense PVA film. An excessive increase in the junction thickness, however, increased both the membrane resistance and the working voltage. When the BPM was alternately sprayed three times with $3 \mathrm{wt} \%$ PVA, $5 \mathrm{wt} \%$ PEI, and $3 \mathrm{wt} \%$ GA solutions, its water splitting-voltage reached the minimum. The membrane voltage was $1.87 \mathrm{~V}$ (error \pm 0.05 ) at a current density of $100 \mathrm{~mA}$

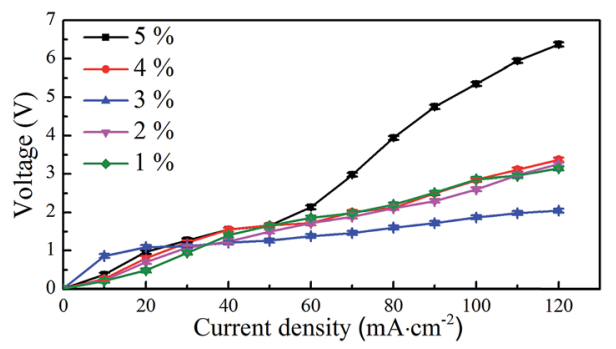

Fig. $9 \quad I-V$ curves of the bipolar membranes with anion-exchange layers prepared with different concentrations of PVA solutions. $\mathrm{cm}^{-2}$. Acid and alkali detected on both sides of the BPM suggested facile ion migration on the membrane.

3.3.4 Effect of spraying cycles. The effect of spraying cycles on the water-dissociation performance of the BPM was depicted in Fig. 10. The CM-ZHJ was sprayed PVA twice, followed by spraying the optimum concentration of 5\% PEI and 3\% GA alternately. When GA and PEI were sprayed alternately once on the CM-ZHJ surface, the AM thus formed was so thin that few functional groups formed, resulting in very weak ion transport. With the increase in number of composite membrane layers, the thickness and density of the membrane also increased, thus increasing the concentration of functional groups and the water dissociation voltage. When the layer was too thick, the rate at which the outer solution diffused into the intermediate layer was low, facilitating the formation of a depletion region. When the number of spraying cycles was excessive, the layers interact electrostatically, thus also affecting ion transfer and the watersplitting of the BPM. The above experimental results therefore indicated that the number of assembled layers could determine the water-dissociation performance of the BPM. When the concentrations of PEI, GA, and PVA were 5, 3, and $3 \mathrm{wt} \%$ respectively, the optimum number of alternate spraying cycles of GA and PEI was three. This corresponded to a water-splitting voltage of $1.82 \mathrm{~V}$ (error \pm 0.05 ) across the BPM at a current density of $100 \mathrm{~mA} \mathrm{~cm} \mathrm{~cm}^{-2}$.

3.3.5 Effect of molecular weight of PEI. Since PEI with low molecular weight possessed few branched chains, it cross-links with GA, forming an anion-exchange layer with few functional groups and weak ability to transfer $\mathrm{OH}^{-}$. Conversely, a higher molecular weight implied more segments in the polymer. High degree of cross-linking of the material could impede hydrolysis of large polymers and thus prevented degradation of the polymer. With its flexible chain segments, PEI with high molecular weight could easily form a dense and smooth anion-exchange layer with abundant dissociating groups and strong ability for ion transfer. When the concentrations of PEI, GA, and PVA were 5,3 , and $3 \mathrm{wt} \%$ respectively, the spraying number of PVA was twice and the alternate spraying of PEI and GA was three times. The decrease in voltage across the BPM showed an improvement of the BPM's water-dissociation performance (Fig. 11). Although the water-splitting voltage of BPM prepared using lowmolecular-weight PEI was low, the acid and alkali on both sides of membrane produced by water dissociation were weak. By contrast, BPM prepared using PEI-7000 showed better

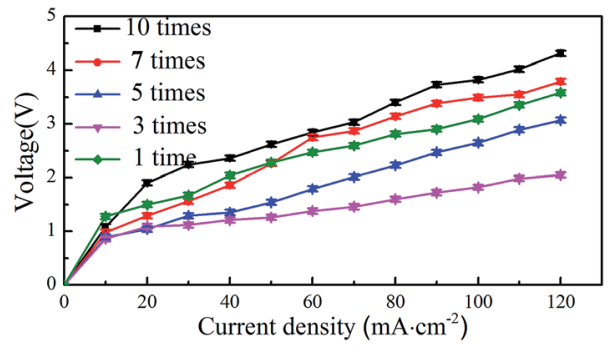

Fig. $10 I-V$ curves of bipolar membranes with anion-exchange layers prepared with different spray times. 


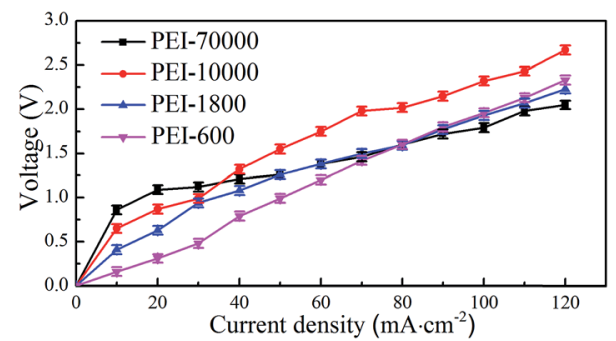

Fig. $11 \quad I-V$ curves of the bipolar membranes with anion-exchange layers prepared with different molecular weights PEI.

performance; its membrane voltage was only $1.79 \mathrm{~V}$ (error \pm 0.05 ) at current density of $100 \mathrm{~mA} \mathrm{~cm}^{-2}$.

\section{Conclusions}

In the present study, the combination of spray assembly with chemical cross-linking technology was successfully used to rapidly fabricate stable high-performance bipolar membranes. The resulting PEI/CM-ZHJ BPM possessed low membrane voltage and shows facile ion migration, which were desirable characteristics of a BPM. The effects of assembly conditions such as polyelectrolyte concentration, cross-linker concentration, composite layers, polyelectrolyte molecular weight, and PVA concentration on the performance of the membrane had been intensively investigated. Under the optimal conditions, the water-splitting voltage of the BPM was $1.79 \mathrm{~V}$ (error \pm 0.05 ) at a current density of $100 \mathrm{~mA} \mathrm{~cm}{ }^{-2}$. Spray cross-linking improved the charge density and ion-exchange capacity of the membrane, as well as enhanced the adhesion between the interfacial layer and anion- and cation-exchange layers. They also modified the BPM microstructure according to the membrane-forming conditions and spraying process parameters. Spray crosslinked assembly was a fast process; it only took several seconds to complete, thus greatly shortening the membrane-forming time and improving the membrane efficiency.

\section{Acknowledgements}

This project was supported by the National Natural Science Foundation of China (Program No. 51473013).

\section{References}

1 B. Bauer, F. J. Gerner and H. Strathmann, Desalination, 1988, 68, 279-292.

2 R. Simons, J. Membr. Sci., 1993, 78, 13-23.

3 K. Nagasubramanian, F. P. Chlanda and K. J. Liu, J. Membr. Sci., 1977, 2, 109-124.
4 M. Reig, C. Valderramaa, O. Giberta and J. L. Cortinaa, Desalination, 2016, 399, 88-95.

5 Y. Li, S. Shi, H. Cao, X. Wu, Z. Zhao and L. Wang, Water Res., 2016, 89, 201-209.

6 C. X. Jiang, Q. Y. Wang, Y. L. Zhang, Y. Li, Y. M. Wang and T. W. Xu, J. Membr. Sci., 2016, 498, 48-56.

7 L. Bazinet, F. Lamarche and D. Ippersiel, Trends Food Sci. Technol., 1998, 9, 107-113.

8 H. Strathmann, J. J. Krola, H. J. Rapp and G. Eigenbergerb, J. Membr. Sci., 1997, 125, 123-142.

9 A. Wang, S. C. Peng, Y. H. Wu, C. H. Huang and T. W. Xu, J. Membr. Sci., 2010, 365, 269-275.

10 J. H. Hao, C. X. Chen, L. Li, L. X. Yu and W. X. Jiang, J. Appl. Polym. Sci., 2001, 80, 1658-1663.

11 T. W. Xu, J. Membr. Sci., 2005, 263, 1-29.

12 J. Balster, S. Srinkantharajaha, R. Sumbharajua, I. Pünta, R. G. H. Lammertinka and D. F. Stamatialisa, J. Membr. Sci., 2010, 365, 389-398.

13 G. Couture, A. Alaaeddine, F. Boschet and A. Bruno, Prog. Polym. Sci., 2011, 36, 1521-1557.

14 C. X. Xu, R. Y. Chen, X. Zheng, X. Chen and Z. Chen, J. Membr. Sci., 2008, 307, 218-224.

15 Q. Y. Wang, B. Wu, C. X. Jiang, Y. M. Wang and T. W. Xu, J. Membr. Sci., 2017, 524, 370-376.

16 J. Pan, L. X. Hou, Q. Y. Wang, Y. B. He, L. Wu, A. N. Mondal and T. W. Xu, Mater. Chem. Phys., 2017, 186, 484-491.

17 K. C. Krogman, J. L. Lowery, N. S. Zacharia, G. C. Rutledge and P. T. Hammond, Nat. Mater., 2009, 8, 512-518.

18 S. Y. Kim, Y. Hong, R. Kavian, S. W. Lee, M. N. Hyder, Y. Shao-Horn and P. T. Hammond, Energy Environ. Sci., 2013, 6, 888-897.

19 A. Alcaraz, P. Ramírez, J. A. Manzanares and S. Mafé, J. Phys. Chem. B, 2001, 105, 11669-11677.

20 H. Fan, D. Q. Shi, H. Yan, S. L. Ji, J. X. Dong and G. J. Zhang, Angew. Chem., Int. Ed. Engl., 2014, 53, 5578-5582.

21 H. W. Fan, N. X. Wang, S. Ji, H. Yan and G. J. Zhang, J. Mater. Chem. A, 2014, 2, 20947-20957.

22 G. J. Zhang, X. Song, N. X. Wang and Z. Z. Liu, J. Membr. Sci., 2008, 325, 109-116.

23 C. C. Feng, J. Xu, M. M. Li, Y. Y. Tang and C. J. Gao, J. Membr. Sci., 2014, 451, 103-110.

24 R. Q. Fu, H. Y. Xue, T. W. Xu and W. H. Yang, J. Colloid Interface Sci., 2005, 285, 281-287.

25 P. Ramirez, H. J. Rapp, S. Reichle, H. Strathmann and S. Mafé, J. Appl. Phys., 1992, 72, 259-264.

26 Y. Wei, C. Li, Y. Wang, X. Zhang, Q. Li and T. W. Xu, Sep. Purif. Technol., 2012, 86, 49-54.

27 H. Meng, Q. Cheng and C. Li, Appl. Surf. Sci., 2014, 303, 399405.

28 J. M. Yang, W. Y. Su and M. C. Yang, J. Membr. Sci., 2004, 236, 39-51. 